\title{
Pneumomediastinum and Cervical Soft Tissue Emphysema as an Important Vital Sign in Hanging: An Autopsy Based Prospective Study
}

\section{Pawan Mittal ${ }^{*}$ and Gaurav Sharma}

Department of Forensic Medicine, B.P.S. Govt. Medical College for Women, Haryana

*Corresponding author: Pawan Mittal, B.P.S. Govt. Medical College for Women, Khanpur Kalan, Sonipat, Haryana, India, Tel: +91-9996031331; E-mail: drmittalpawan@gmail.com

Received date: February 13, 2017; Accepted date: March 07, 2017; Published date: March 11, 2017

Copyright: (C) 2017 Mittal P, et al. This is an open-access article distributed under the terms of the Creative Commons Attribution License, which permits unrestricted use, distribution, and reproduction in any medium, provided the original author and source are credited.

\begin{abstract}
Objective: To determine the frequency and reliability of cervical soft tissue emphysema and pneumomediastinum in autopsy cases of hanging.

Material and Method: We prospectively studied 30 autopsy cases of hanging including 18 males and 12 females with age ranging from 19 to 65 years. The control group consisted of age and sex matched 30 cases that died due to various natural pathologies. Along with this, a small group of five cases consisting of homicidal asphyxial deaths from ligature and manual strangulation was also studied for this purpose. Various other known vital phenomenon in hanging were also recorded in the form of hemorrhages in the subcutaneous tissues, laryngeal hyoid skeleton fractures, conjunctival and facial petechial hemorrhages, hemorrhages over clavicular origin of sternocleidomastoid muscle, carotid intimal tears (Amussat's sign), Simon's bleeding and hemorrhages in the respiratory auxiliary muscles of respiration. The dissection and preparation of the neck was done in layers. None of the cases underwent cardiopulmonary resuscitation. Signs of putrefaction were absent.
\end{abstract}

Results: The soft tissue emphysema was seen in 22 cases (73.3\%) of hanging as fine soapy air bubbles in the mediastinum that could be traced further in the superficial and deep tissues of the neck up to the level of ligature mark beyond which it was missing. Among two other groups, 3 controls depicted this finding while no traces of emphysema were seen in any of the strangulation cases.

Conclusion: Cervical soft tissue emphysema along with pneumomediastinum is a frequent and important vital finding in cases of death due to hanging. Whenever possible, the vitality of the sign should be considered in conjunction with other known vital signs of hanging. However a proper care should be taken during dissection of cervical and thoracic structures to avoid artefactual introduction of air in the soft tissues. The interpretation must be based on sound medical grounds and observations.

Keywords: Hanging; Emphysema; Pneumomediastinum; Sternocleidomastoid; Air; Autopsy

Abbreviations: CSTE: Cervical soft tissue emphysema; CPR: Cardiopulmonary resuscitation

\section{Introduction}

Pneumomediastinum or mediastinal emphysema implies the presence of air in the mediastinum. It is a well-known phenomenon in the clinical practice where it has been described in multiple conditions causing alveolar rupture such as Valsalva maneuver, parturition, postoperative vomiting, acute exacerbation of bronchial asthma [1-4]. The clinical diagnosis of the mediastinal and soft tissue emphysema is primarily based upon radiological findings. There is a paucity of forensic literature highlighting the autopsy demonstration of such emphysema. Limited forensic studies are available that have shown this finding to be a vital determinant in making the conclusion of hanging at autopsy $[5,6]$. Postmortem imaging has demonstrated the finding of pneumomediastinum and CSTE, with gas collection and air following visceral spaces around trachea, oesophagus, large vessels of the neck and even the cervical spinal canal in cases of hanging using MSCT and
MRI protocols [6,7]. The proposed mechanism for this finding includes either direct or indirect trauma to the cervical airways because of ligature pressure and stretching, or rupture of terminal alveoli caused by elevated intra-thoracic pressure with subsequent dissection of air in the mediastinal and cervical soft tissues around visceral structures $[8,9]$. The latter mechanism is known as Macklin effect [10]. The aim of this study was to determine the frequency of pneumomediastinum and CSTE at autopsy in cases of deaths due to hanging.

\section{Material and Methods}

A prospective study was carried out that included 30 cases of suicidal hanging that were brought for autopsy to our forensic medicine department over a period from October 2015 to August 2016. Detailed information of the circumstances was gained from police agency, enquiry from relatives and witnesses, history of previous suicidal attempts and suicidal notes. A visit to the death scene was also made in few cases, wherever deemed necessary. The sample included 18 males and 12 females with ages ranging from 19 to 65 years. The cases consisted of both complete and partial and typical and atypical hangings. In the latter instance there were no cases classified as 
Page 2 of 6

anterior hanging, i.e., with knot over the submental mandibular region. The knot site was determined from the crime scene photographs and course of ligature mark at autopsy. All the cases were declared dead on arrival in the hospital emergency unit without any cardiopulmonary resuscitation or treatment being carried out. No additional trauma was present in any of the cases.

The post mortem interval was between 12 to 24 hours, determined on the basis of postmortem

changes and information regarding when the victim was last seen alive. Toxicology examination of the viscera and body fluids did not reveal ethyl alcohol or any other common poison in any subject. The histopathological examination of organs did not show any macroscopic or microscopic evidence of chronic or significant pathology of the respiratory tract or other organs. The dissection and preparation of the neck was done in layers as described in the forensic literature [11]. The skin, subcutaneous tissues, muscles and deep structures of the neck were dissected and reflected in layer-wise manner keeping traction at the minimum, to avoid artefactual sucking of air in the tissues. The mediastinal and CSTE was recorded and stated to be positive by finding an almost continuous stream of fine soapy air bubbles in the superficial and/or deep soft tissues of the mediastinum and neck. This could be easily observed in most cases over the pulmonary viscera, and around the trachea, oesophagus, neck vessels and dorsal surface of the upper layer strap muscles and ventral aspect of deep strap muscles.

Other vital findings of hanging were recorded as hemorrhages in the subcutaneous tissues, laryngeal hyoid skeleton fractures, conjunctival and facial petechial hemorrhages, hemorrhages over the clavicular origin of sternocleidomastoid muscle, carotid intimal tears (Amussat's sign), Simon's bleeding and hemorrhages in the respiratory auxiliary muscles of respiration, so that along with one or more of these findings, the soft tissue emphysema was considered to be vital in origin. An oblique ligature mark with typical appearance consistent with knot site was present in each case.

A group of five cases of deaths from homicidal asphyxia were also autopsied during this period and evaluated for similar evidence of soft tissue emphysema. The cases consisted of two deaths from ligature strangulation, two cases of manual strangulation and one involving both ligature and manual strangulation with blunt head trauma. All cases were young males in their 30's. There was no evidence of chest trauma in any case. No CPR was performed and putrefaction was absent. Layer-wise dissection of the neck was performed in each case.

A control group of 30 non-hanging age and sex matched cases (age range 19 to 65 years; 18 males and 12 females) was formed who died from a variety of natural cardiac and non-cardiac causes. The causes of death included: 12 cases of complicated coronary atherosclerosis, six cases belonged to acute left ventricular failure, four young adults died of cardiomyopathy and myocarditis, four cases of hypertensive intracranial hemorrhage, three from bleeding peptic ulcer and one from bleeding oesophageal varices. The basic methodology and criteria of selection for these cases were the same as the hanging cases. All were declared dead on arrival in the emergency unit without any cardiopulmonary resuscitation being carried out. The cases lack any signs of initial putrefaction, the postmortem interval was between 12 to 24 hours and toxicology screening did not reveal ethanol or any other common poison. There was no autopsy evidence of trauma in any of the subjects. The dissection and preparation of the neck was done in layers and the same autopsy technique, as for hanging cases, was used for all these cases.
The finding of CSTE and pneumomediastinum was confirmed by two independent observers.

\section{Results}

CSTE and pneumomediastinum was seen in 22 out of 30 cases (73.3\%) of hanging. The finding was found to be independent of the age and sex of the individual. The presence of CSTE and pneumomediastinum was not dependent upon the type of hanging and suspension point. As we did not get any case belonging to anterior hanging, any contribution of the respiratory asphyxia to the proposed carotid artery occlusion/carotid sinus related vagal death could not be evaluated [12]. In the neck the air bubbles could be well appreciated up to the level of mark (at lower margin) above which they were missing (Figure 1).

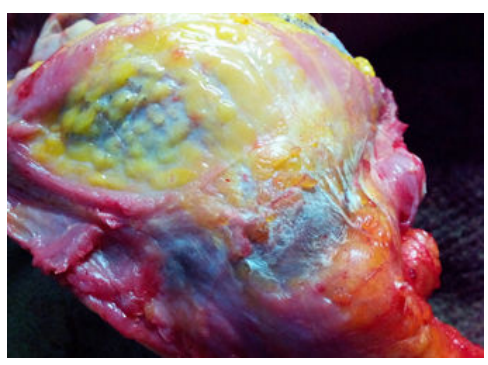

Figure 1: Air bubbles at the level of mark (at lower margin).

A further evaluation for the specific location of emphysema showed that CSTE and pneumomediastinum occur as coexistent findings, as none of the cases revealed these as independent findings which also suggest the presence of a pressure gradient between ruptured alveoli and the upper respiratory tract. A correlation of the phenomenon was made with Simon's bleeding (13/30 positive $43.3 \%)$ and clavicular periosteal hemorrhage (18/30 positive- $60 \%)$ at the sternocleidomastoid muscle origin.

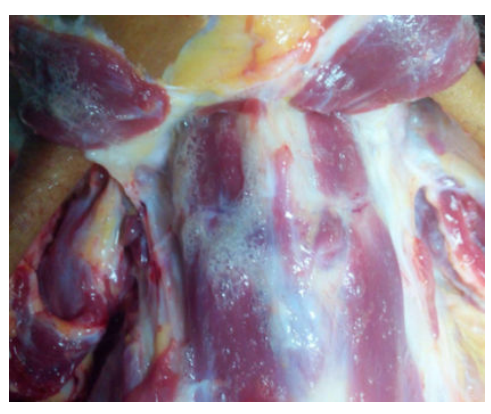

Figure 2: Air bubbles at the clavicles near the root of the neck and on the dorsal surface of upper layer strap muscles and ventral surface of deep strap muscles.

Soft tissue emphysema with Simon's bleeding was found in 9 of 13 cases $(69.23 \%)$ while the coexistence with clavicular periosteal hemorrhage was seen in 13 of 18 cases (72.22\%).

In the neck the air bubbles were found to be prominent over the clavicles near the root of the neck and on the dorsal surface of upper 
Citation: Mittal P, Sharma G (2017) Pneumomediastinum and Cervical Soft Tissue Emphysema as an Important Vital Sign in Hanging: An Autopsy Based Prospective Study. J Forensic Res 8: 1000371. doi:10.4172/2157-7145.1000371

Page 3 of 6

layer strap muscles and ventral surface of deep strap muscles, after reflecting them (Figure 2).

In the neck muscles, the dorsum aspect of sternocleidomastoid muscle depicted this finding most frequently (Figure 3). Pneumomediastinum was prominent in midline mediastinal soft tissues (Figure 4).

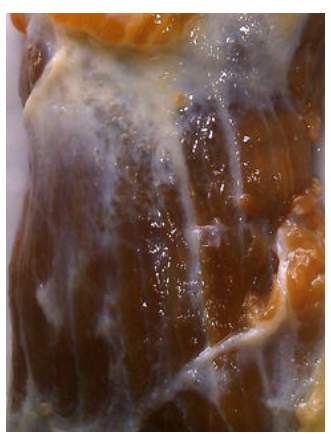

Figure 3: The dorsum aspect of sternocleidomastoid muscle.

Furthermore the evidence of emphysema could be well appreciated during autopsy from the crackling sound under the dissection knife. The autopsy did not reveal any mucosal lesions of either the upper or lower airways. All the findings were noted and photographed in situ as well as after separating them from their anatomical attachments.

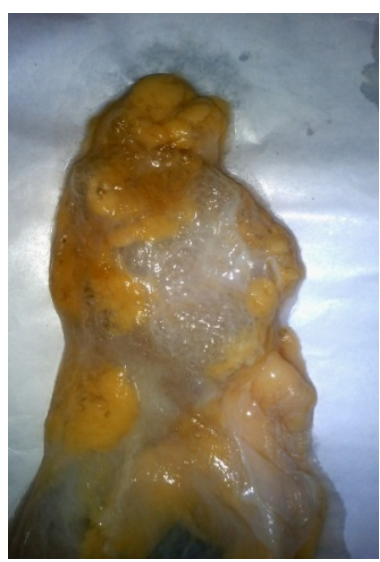

Figure 4: Pneumomediastinum in midline mediastinal soft tissues.

In five strangulation cases, toxicology analysis revealed a blood alcohol concentration of $65 \mathrm{mg} \%$ and $110 \mathrm{mg} \%$ in one case of manual strangulation and one case of strangulation with head trauma respectively. Typical findings consisted of showers of petechial hemorrhages and congestion syndrome above the level of neck compression, hyposphagma, strap muscles hemorrhages and fractures of the laryngeal framework.

Evidence of any cardiopulmonary pathology was ruled out from gross and microscopic histopatholgical examination. Layer-wise and careful dissection of the neck and chest did not reveal any evidence of pneumomediastinum (Figure 5), CSTE (Figure 6) or air bubbles around mark of strangulation (Figure 7).

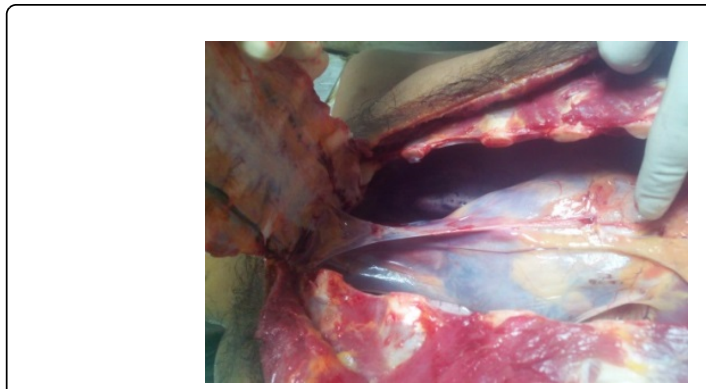

Figure 5: Dissection of the neck and chest.

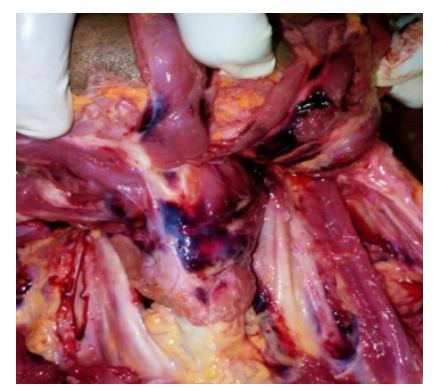

Figure 6: Cervical soft tissue emphysema.

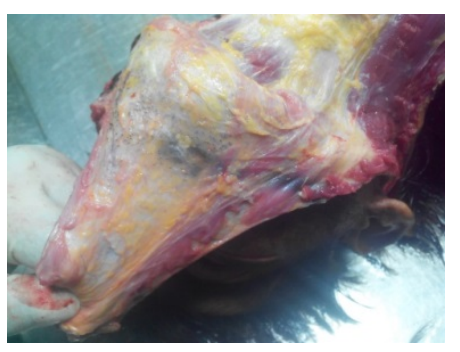

Figure 7: Air bubbles around mark of strangulation.

CSTE and pneumomediastinum was recorded in three control cases (10\%); two cases of acute LVF and one case of hypertensive cerebral hemorrhage. However it was neither too gross nor present as a continuous stream. A patchy, irregular and focal distribution of separate tiny air bubbles was present at multiple foci in all three cases, which could be easily differentiated from continuous streaming emphysema found in hangings.

\section{Discussion}

Hussarek and Wolf made the first radiology-based clinical observation of CSTE in cases of attempted strangulation [13]. Canizares et al. reported a case of attempted hanging with subcutaneous emphysema [14]. In the clinical practice pneumomedisatinum and CSTE is commonly observed in cases of attempted strangulations where victims survived, were admitted and underwent radiological examination. The detection of the gas as pneumomedisatinum and/or CSTE has been infrequently demonstrated at autopsy which is evident from the scarce reporting of 
this finding in deaths due to strangulations $[5,6]$ and in other cases also. In the latter instance, Byard has described a case of death due to chronic obstructive pulmonary disease with pneumothorax and subcutaneous emphysema in which widespread and massive emphysema was demonstrated at autopsy in the form of air bubbles, all over the upper torso region. The emphysema was dissecting into the dermis and fascia of the muscles such as sternocleidomastoid [15].

A kind of frothy air, soap bubble-like formation in cervical soft tissue can be an artefactual phenomenon resulting from the stretching and exenterating of the neck organs during autopsy. To avoid this and differentiate it from CSTE in cases of hanging, it is important to dissect the neck in layer-wise manner, keeping the traction at minimum while preparing neck muscles and soft tissues [11]. The muscles are cut from their bony attachments and examined on their dorsal surface for any evidence of emphysema that appears as fine air bubbles. The ligature pressure makes a kind of binding for these bubbles so that the emphysema is not appreciable beyond the ligature mark [5].

In the past few years, postmortem imaging has emerged as an effective tool in elucidating the important ante mortem findings prior to the commencement of autopsy. Postmortem multi-slice computed tomography (MSCT) and magnetic resonance imaging (MRI) have been the constant and regular procedures carried out in some developed nations to know the inside story of the corpse prior to dissection [16,17]. The cross sectional radiological imaging methods used in clinical medicine have been implemented in the field of forensic medicine which have revolutionized forensic case work. Although the exact value of postmortem imaging in detecting specific conditions has not been fully established, in specific cases, however, it can be used as a diagnostic tool demonstrating findings that remain undetected during autopsy. Aghayev et al. used postmortem imaging with MSCT and MRI, to demonstrate pneumomediastinum and soft tissue emphysema of the neck in three cases of suicidal hanging. Although the soft tissue emphysema was well appreciated in all three cases on radiological imaging, on autopsy two cases were completely lacking this finding [6]. Similarly, in a case described by SieswerdaHoogendoorn et al., pneumomediastinum and soft tissue emphysema were detected with postmortem CT scan in a 3-year-old girl who died due to hanging. However, it was not found subsequently during autopsy [7]. The reasons for these discrepancies might be the variations in dissection techniques and/or inter-individual observation differences. However, these cases demonstrate one of the added values of postmortem imaging, the possibility of demonstrating findings that remain undetected during autopsy.

The underlying mechanism of pneumomediastinum and CSTE in hanging is not completely understood. Two pathophysiological mechanisms have been proposed to explain the presence of gas in the soft tissues. First, a traumatic direct or indirect tear or rupture of lower or upper airways might result from local violence from ligature pressure [6]. The other mechanism involves an increase in intrapulmonary pressure during physical straining causing pulmonary interstitial emphysema [18]. This mechanism was further confirmed by Macklin and Macklin who proposed rupture of terminal alveoli caused by a sudden increase in intra-thoracic pressure due to forceful expiration against a closed glottis during hanging. There is subsequent dissection of air along the visceral spaces around trachea, bronchi, oesophagus and large vessels in the mediastinum and further up in the neck into superficial and/or deep soft tissues. This is the well accepted mechanism at present, also known as Macklin effect [10]. A pressure gradient is formed between the site of alveolar rupture and obstruction zone in the neck, leading to the formation and dissection of air bubbles in the mediastinal and neck soft tissues. Pneumomediastinum and CSTE arise in various conditions because of the Macklin Effect, as a result of an alveolar rupture secondary to high intra-alveolar pressure $[8,9]$. Both mechanisms are compatible with the finding of air in the visceral space around the trachea, esophagus and big vessels of the neck, the structures serving as guides for air spread. Cases of airway mucosal injuries with complete disruption, including decapitation and transverse laceration of upper airways, have been reported in hanging deaths $[19,20]$. Gill et al. have reported two cases of crico-tracheal disruption owing to hanging which lead to the development of local subcutaneous emphysema [21].

We did not find mucosal injury to either the upper or the lower airway in any of the cases so the first mechanism is unlikely to be responsible for soft tissue emphysema in this study. The Macklin effect is most likely the responsible mechanism for soft tissue air entry in our cases. These findings are in accordance with previous studies $[5,6]$. However, recent studies on filmed hangings have suggested that there might not even be fatal airway obstruction in hanging [22]. This largely undermines the effects of complete glottic closure and increased intrathoracic pressure, thereby creating doubts over the true existence of the Macklin effect in hanging. The genuineness of the phenomenon is further questionable based on reports of hanging deaths taking place in individuals with ligature high up in the neck and a permanent tracheostomy opening below $[23,24]$. However, in the authors' opinion, it is possible that some alternative mechanism of death, in place of respiratory asphyxia, might be operating in the cases lacking this finding. So the pneumomedisatinum and CSTE might not be exclusively found in all cases of hanging. As there has been no mentioning of the knot site and typical-atypical hanging in the videofilmed hanging cases, an exact mechanism of death in those cases cannot be explained, which is frequently dependent on the mode of ligature application and knot site. The involvement of neck vital structures in hanging is also dependent on the amount of weight force acting on the neck which is multifactorial in origin and is different for individual vital neck structures [12]. Furthermore it cannot be emphasized enough that death in hanging is primarily caused by compression of the blood vessels of the neck, thereby causing an insufficient amount of oxygenated blood reaching the brain. Blockage or compression of the air passages is not necessary to cause death in hanging [24]. Findings of cerebral anaemia and hypoxia cannot be seen at autopsy as the death in hanging occurs in too short time interval to make these changes apparent. Although if the victim survives, ischaemic infarcts in the classical watershed zones of the brain may be demonstrated grossly as well as histologically [25]. Therefore, it is not often possible to comment with certainty which mechanism might be predominantly operating in a particular case. Also, unlike postmortem imaging, autopsy does not allow for the perfect detection of the track followed by the gas molecules along chest and neck organs in such cases. Soft and solid neck structures preparation by layers only allows for the visualization of CSTE, but does not explain its origin clearly. Therefore, the patho-physiology of the finding remains unclear most of the time.

Cases of non-traumatic pneumomediastinum and cervical emphysema have been extensively reported in the living individuals including cases of inhalational drug abuse [1], postoperative vomiting [3] and also as a spontaneous phenomenon [26]. A case of spontaneous pneumomediatinum in a trombonist has been described by Ito et al. The authors proposed the mechanism as repetitive overinflation of lungs with alveolar tenderness and high intra-alveolar 
Page 5 of 6

pressure causing alveolar rupture [27]. Histological demonstration of pulmonary micro-morphology was made by Grellner and Madea in 106 cases of fatal strangulation and hanging. The authors recorded perivascular and intra-alveolar hemorrhages, local dystelectasis and focal emphysema as frequent histological findings in such cases [28].

Numerous vital findings have been described in connection with hanging. Important among these are hemorrhagic zone between ligature marks, periosteal hemorrhages at the clavicular origin of sternocleidomastoid muscle, hemorrhages into the back and auxiliary muscles of respiration, bowel wall hemorrhages, Simon's bleedings and carotid intimal tears (Amussat's sign). These vital reactions are not compulsory in hanging, and they can also be produced postmortem [29]. Along with these, a few controversial findings of doubtful significance have been described in the literature [30,31]. In contrast, pneumomediastinum and cervical emphysema are not the products of circulation, rather they result from attempted breathing against closed glottis due to mechanical obstruction; therefore, they are more reliable.

None of our five cases of deaths due to ligature and manual strangulation revealed any air bubbles in either mediastinum or higher cervical soft tissues. This may be explained by the fact that the mechanism of death predominantly involved in such cases is either jugular venous and/or carotid artery occlusion or sudden vagus induced cardiac arrest. Furthermore the amount of pressure required to compress the trachea and/or to achieve glottis closure is usually not achieved from manual means of strangulation. This is in contrast to hanging where comparably more neck constriction exists making tracheal and glottis closure relatively easier [12]. At first instance, the results and the existing mechanisms make the finding of emphysema quite specific for hanging, especially for cases where the potential confusion exists between hanging and strangulation. But we cannot firmly conclude this finding for cases of strangulation due to the small size of our sample. A larger study involving deaths from homicidal asphyxial means is required for such a firm opinion.

In other cases of mechanical asphyxial deaths, such as smothering, choking, hangings with soft broad ligatures etc., where the obvious injuries to the superficial as well as deep neck structures may be absent, presence of cervical soft tissue emphysema and pneumomediastinum may act as a useful vital parameter in suggesting fatal airway obstruction.

\section{Conclusion}

The finding of pneumomediastinum and CSTE at autopsy can be important and reliable vital evidence in cases of hanging, especially when the circumstances are non-explanatory. However, it is important to rule out any evidence of incipient putrefaction, cardiopulmonary resuscitation or already existing cardiac or pulmonary pathology before making an opinion of hanging from this finding. Whenever possible, the vitality of the sign should be considered in conjunction with other known vital signs of hanging. Furthermore a layer by layer and bloodless dissection of the neck should be strictly followed in such cases, as artefacts may be introduced at any stage if proper precautions are not taken, which may spoil the vital findings significantly. The underlying mechanism of the soft tissue emphysema is not completely understood, so the interpretation must be made with caution, based on sound medical grounds.

\section{References}

1. Miller WE, Spiekerman RE, Hepper NG (1972) Pneumomediastinum resulting from performing Valsalva maneuvers during marihuana smoking. Chest 62: 233-234.

2. Maunder RJ, Pierson DJ, Hudson LD (1984) Subcutaneous and mediastinal emphysema. Pathophysiology, diagnosis, and management. Arch Int Med 144: 1447-1453.

3. Bremner WG, Kumar CM (1993) Delayed surgical emphysema, pneumomediastinum and bilateral pneumothoraces after postoperative vomiting. Br J Anaesth 71: 296-297.

4. Akinyemi RO, Ogah OS, Akisanya CO, Timeyin AO, Akande KO, et al. (2007) Pneumomediastinum and Subcutaneous Emphysema Complicating Acute Exacerbation of Bronchial Asthma. Ann Ib Postgrad Med 5: 78-79.

5. Nikolić S, Zivković V, Babić D, Juković F (2012) Cervical soft tissue emphysema in hanging--a prospective autopsy study. J Forensic Sci 57: 132-135.

6. Aghayev E, Yen K, Sonnenschein M, Jackowski C, Thali M, et al. (2005) Pneumomediastinum and soft tissue emphysema of the neck in postmortem CT and MRI; a new vital sign in hanging? Forensic Sci Int 153: 181-188.

7. Sieswerda-Hoogendoorn T, Strik AS, Hilgersom NF, Soerdjbalie-Maikoe V, Van Rijn RR (2014) Pneumomediastinum and soft tissue emphysema in pediatric hanging. J Forensic Sci 59: 559-563.

8. Wintermark M, Schnyder P (2001) The Macklin effect-a frequent etiology for pneumomediastinum in severe blunt chest trauma. Chest 120: 543-547.

9. Sakai M, Murayama S, Gibo M, Akamine T, Nagata O (2006) Frequent cause of the Macklin effect in spontaneous pneumomodiastinumdemonstration by multidetector-row computed tomography. J Comput Assist Tomogr 30: 92-94.

10. Zylak CM, Standen JR, Barnes GR, Zylak CJ (2000) Pneumomediastinum revisited. Radiographics 20: 1043-1057.

11. Vanezis P (1993) Post mortem techniques in the evaluation of neck injury. J Clin Pathol 46: 500-506.

12. Saukko P, Knight B (2016) Knight's Forensic Pathology. (4th edn), CRC Press, Taylor \& Francis Group, Boca Raton, London, New York.

13. Hussarek M, Wolf G (1971) Subcutaneous emphysema of neck and larynx following attempted strangulation. Z Rechtsmed 68: 41-44.

14. Canizares MA, Arnau A, Fortea A, Zarzuela V, Martinez-Vallina P, et al. (2000) Hyoid fracture and traumatic subcutaneous cervical emphysema from an attempted hanging. Apropos a case. Arch Bronconeumol 36: 52-54.

15. Byard RW (2016) Gross and microscopic features of extensive subcutaneous emphysema. Forensic Sci Med Pathol 12: 372-375.

16. Thali MJ, Yen K, Schweitzer W, Vock P, Boesch C, et al. (2003) Virtopsy, a new imaging horizon in forensic pathology: virtual autopsy by postmortem multislice computed tomography (MSCT) and magnetic resonance imaging (MRI)--a feasibility study. J Forensic Sci 48: 386-403.

17. Thali MJ, Yen K, Plattner T, Schweitzer W, Vock P, et al. (2002) Charred body: virtual autopsy with multi-slice computed tomography and magnetic resonance imaging. J Forensic Sci 47: 1326-1331.

18. Joannides M, Tsoulos GD (1930) The etiology of interstitial and mediastinal emphysema. Arch Surg 121: 333-339.

19. Tattoli L, Buschmann CT, Tsokos M (2014) Remarkable findings in suicidal hanging. Forensic Sci Med Pathol 10: 639-642.

20. Hejna P, Bohnert M (2012) Decapitation in suicidal hanging-vital reaction patterns. J Forensic Sci 58: S270-S277.

21. Gill SS, Singh CM, Eggleston FC (1977) Cricotracheal disruption owing to strangulation. A report of two cases with successful surgical repair. J Thorac Cardiovasc Surg 73: 948-950.

22. Sauvageau A, Laharpe R, King D, Dowling G, Andrews S, et al. (2011) Agonal sequences in 14 filmed hangings with comments on the role of the 
Citation: Mittal P, Sharma G (2017) Pneumomediastinum and Cervical Soft Tissue Emphysema as an Important Vital Sign in Hanging: An Autopsy Based Prospective Study. J Forensic Res 8: 1000371. doi:10.4172/2157-7145.1000371

Page 6 of 6

type of suspension, ischemic habituation, and ethanol intoxication on the timing of agonal responses. Am J Forensic Med Pathol 32: 104-107.

23. Cooke CT, Cadden GA, Hilton JMN (1989) Unusual hanging deaths. Am J Forensic Med Pathol January 9: 277-282.

24. DiMaio VJ, DiMaio D (2001) Forensic Pathology. Practical aspects of criminal and forensic investigations. (2nd edn), CRC Press, Boca Raton London, NewYork Washington, USA.

25. Oehmichen M, Auer RN, Konig HG (2006) Forensic Neuropathology and Associated Neurology. (1st edn), Springer, Berlin Heidelberg, NewYork, US.

26. Abolnik I, Lossos IS, Breuer R (1991) Spontaneous pneumomediastinum. A report of 25 cases. Chest 100: 93-95.
27. Ito S, Takada Y, Tanaka A, Ozeki N, Yazaki Y (1990) A case of spontaneous pneumomediastinum in a trombonist. Kokyu to junkan. Respiration \& circulation 37: 1359-1362.

28. Grellner W, Madea B (1994) Pulmonary micrornorphology in fatal strangulations. Forensic Sci Int 67: 109-125.

29. Puschel K, Turk E, Lach H (2004) Asphyxia-related deaths. Forensic Sci Int 144: 211-214.

30. Zhu BL, Ishida K, Fujita MQ, Maeda H (2000) Immunohistochemical investigation of pulmonary surfactant in fatal mechanical asphyxia. Int $J$ Legal Med 113: 268-271.

31. Fieguth A, Albrecht UV, Bertolini J, Kleemann J (2003) Intracartilaginous haemorrhagic lesions in strangulation? Int J Legal Med 117: 10-13. 